\title{
Nedocromil sodium versus sodium cromoglycate in treatment of exercise-induced bronchoconstriction: a systematic review
}

\author{
K.D. Kelly*, , C.H. Spooner, ${ }^{\#, \text { B.H. Rowe }}$
}

\begin{abstract}
Nedocromil sodium versus sodium cromoglycate in treatment of exercise-induced bronchoconstriction: a systematic review. K.D. Kelly, C.H. Spooner, B.H. Rowe. (C) ERS Journals Ltd 2001.

ABSTRACT: The objective of this review was to compare the effects of prophylactic doses of nedocromil sodium (NCS) and sodium cromoglycate (SCG) on postexercise lung function, in persons diagnosed with exercise-induced bronchoconstriction.

Randomized controlled trials were identified from the Cochrane Airways Review Group Asthma Register, plus hand searching for trials in journals, bibliographies of relevant studies and review articles. Randomized controlled trials comparing NCS to SCG in prophylactic treatment of exercise-induced bronchoconstriction were eligible. Studies were pooled using odds ratios (OR) for dichotomous outcomes or weighted mean differences (WMD) with 95\% confidence intervals $(95 \% \mathrm{CI})$ for continuous outcomes.

No significant differences were noted between NCS and SCG with respect to the maximum per cent decrease in forced expiratory volume in one second (WMD=-0.88; 95\% CI -4.50-2.74), complete protection (OR=0.95; 95\% CI 0.50-1.81), clinical protection (OR=0.71; 95\% CI 0.36-1.39), unpleasant taste $(\mathrm{OR}=6.85 ; 95 \%$ CI $0.77-60.73)$, or sore throat $(O R=3.46 ; 95 \%$ CI $0.32-37.48)$. Subgroup analyses based on age, dosages of medications and timing of exercise postinhalation were consistent with the overall pooled analyses.

No significant differences were evident between the effects of nedocromil sodium and sodium cromoglycate during the immediate postexercise period in adults and children with exercise-induced bronchoconstriction, with regards to maximum per cent decrease in forced expiratory volume in one second, complete protection, or clinical protection. Side-effect profiles were similar.

Eur Respir J 2001; 17: 39-45.
\end{abstract}

Airway hyperreactivity leading to airway narrowing following an exercise challenge is a phenomenon known as exercise-induced bronchoconstriction (EIB). It occurs in $70-80 \%$ of subjects with asthma [1] and an estimated $12-15 \%$ of the general population [2]. EIB is characterized by a transient decrease in the forced expiratory volume in one second (FEV1) or the peak expiratory flow rate (PEFR) provoked by $6-15 \mathrm{~min}$ of continuous, strenuous exercise [3]. By consensus, postexercise decreases of $10-20 \%$ in FEV1 or PEFR indicate mild EIB, falls of $20-40 \%$ represent moderate EIB, and $>40 \%$ represents severe EIB $[4,5]$. This airflow obstruction causes dyspnoea, cough, wheeze, premature fatigue, and prolonged recovery time. Maximum bronchoconstriction typically occurs $5-15 \mathrm{~min}$ following exercise and usually subsides spontaneously within 20-60 min [6]. The severity and impact of symptoms is dependent on several factors: concomitant asthma therapy, intensity and duration of activity, environmental conditions, degree of underlying bronchial hyperreactivity, level of physical conditioning, and the time interval since previous exercise [7].

\begin{abstract}
*Dept of Rural Health, Faculty of Medicine, Dentistry and Health Sciences, University of Melbourne, Melbourne, Australia, "Division of Emergency Medicine, Faculty of Medicine and Dentistry, University of Alberta, Canada. Airway Review Group, Cochrane Collaboration, London, UK.
\end{abstract}

Correspondence: B.H. Rowe, Division of Emergency Medicine, 1G1.63 Walter McKenzie Health Centre, 8440-112 St. Edmonton, AB, Canada, T6G 2B7. Fax: 7804073314

Keywords: Bronchoconstriction, cromolyn sodium, exercise-induced asthma, forced expiratory volume, nedocromil

Received: November 251999

Accepted after revision July 122000

This systematic review was partially funded by the Division of Emergency Medicine at the University of Alberta.

A version of this review is published in an electronic format in the Cochrane Library.
Management of EIB has been the focus of intense pharmacotherapeutic research and the emphasis has been on prophylactic therapy. Different drugs have proven useful; however, there remains considerable debate regarding the merits, optimal dose and appropriate method of delivery for each treatment. Traditionally, inhaled $\beta$-agonists and other bronchodilating agents have been the drugs of choice [6, 8]. Recently, anti-inflammatory agents, such as nedocromil sodium (NCS), sodium cromoglycate (SCG), and inhaled corticosteroids (ICS) have been evaluated [9]. SCG inhibits mast cell degranulation, and can prevent or attenuate bronchospasm induced by exercise or cold air, particularly in children. NCS is a chemically unrelated drug with similar effects [10]. NCS has been reported to be effective against bronchospasm induced by antigens, fog, cold air, sulphur dioxide, and exercise [11]. A recent systematic review comparing NCS to placebo established this drug's efficacy in attenuating EIB [9]. Although, individual randomized controlled trials (RCTs) have been conducted to compare NCS with SCG, to date no systematic review that combines all trials to 
obtain a pooled estimate of the difference in the magnitude of effect between these drugs has been published.

This systematic review examines the available evidence from RCTs, comparing the prophylactic efficacy of NCS versus SCG in preventing or attenuating EIB. The objective of this review was to quantitatively compare the effects of NCS and SCG administered by a pressurized aerosol or metered dose inhaler (MDI) prior to a strenuous exercise challenge in subjects who suffer from EIB. Specifically, the effects of NCS and SCG were examined from studies that compare the two treatments directly.

\section{Materials and methods}

\section{Inclusion criteria}

Articles that satisfied all of the following criteria were searched for:

Design. Reported studies had to be RCT or quasi RCTs.

Populations. Prior to entry into the trial, participants had to have a diagnosis of EIB. This meant they had to demonstrate a reproducible fall in FEV1 of $\geqslant 10 \%$ postexercise on a control challenge with no pretreatment. The maximum per cent fall in FEV1 or PEFR postexercise was used to define EIB severity: 10-20\%, mild; $20-40 \%$, moderate; and $>40 \%$, severe $[1,4,6]$. Studies which did not state specific criteria for EIB or that included people with impairment $<10 \%$ were excluded. Studies recruiting children $(\geqslant 6$ $17 \mathrm{yrs})$, or adults $(\geqslant 18 \mathrm{yrs})$ were included and these age designations formed one of the a priori subgroup analyses.

Interventions. Studies reporting results from participants who were randomized to receive a single prophylactic dose of either inhaled NCS or SCG prior to a standardized exercise challenge were included. Studies that had additional drug arms were considered, but only results pertaining to NCS and SCG were included in this review. Studies that involved delivery using nasal sprays were not included.

Outcomes. All patient outcomes, both subjective and objective, were considered. The primary outcomes of interest were continuous data from pulmonary function measures (FEV1 and PEFR). The conventional method to quantify EIB is to measure the maximum reduction in FEV1 or PEFR and to express it as a per cent fall index, that is, to express the reduction in lung function postexercise as a per cent of the preexercise value. The authors also wanted to compare the drugs on three secondary outcomes that were of interest: a) complete protection from EIB, b) clinical protection, and c) the number and nature of adverse effects experienced.

A measure of whether or not complete protection was achieved was determined at two levels: 1) if the maximum percentage drop in FEV1 postexercise was not within the normal range (i.e. remained $>10 \%$ ); and
2) if the drop remained greater than the diagnostic cutoff (i.e. remained $>15 \%$ ) complete protection was recorded as not achieved. A drug does not offer significant clinical protection if the percentage fall after receiving the active drug is less than half the percentage drop after receiving a placebo (i.e. $<50 \%$ improvement) $[5,12,13]$.

\section{Search strategies for identifying studies}

The Cochrane Airways Review Group (ARG) developed an "Asthma and Wheez* RCT" database through a comprehensive search of EMBASE (1980present), MEDLINE (1966-present), and CINAHL (1982-present). In addition, hand searching of 20 respiratory care journals have been completed and relevant articles included in the registry. Finally, the register was regularly updated with searches of the Cochrane Clinical Trial Register (CCTR). The current overview includes ARG register updates to August 1999.

Firstly, a search of this database was completed using the following terms: 1) Asthma OR Wheez* AND 2) exercise OR exercise induced AND 3) Nedocromil OR Nedocromil Sodium OR Tilade OR NS AND 4) Cromolyn Sodium OR Sodium Cromoglycate OR SCG OR CS OR CR. Randomized controlled trials were identified in the register using the following search strategy: (placebo* OR trial* OR random* OR doubleblind OR double blind OR single-blind OR single blind OR controlled study OR comparative study) [14]. The ARG register contains studies published in foreign languages, and trials were not excluded on the basis of language. When necessary, attempts were made to translate articles from the foreign language. Secondly, reference lists of each primary study and review article were checked to identify additional potentially relevant citations. Thirdly, inquiries regarding other published or unpublished studies known and/or supported by the authors of the primary studies were made. Fourthly, personal contact was made with colleagues, collaborators and other investigators working in the field of asthma in order to identify potentially relevant studies. Finally, the pharmaceutical company which manufacturers both NCS and SCG (Rhône-Poulenc Rorer Ville St-Laurent, Quebec, Canada) was contacted to solicit additional citations. Published and unpublished studies were eligible for inclusion.

From the title, abstract, or descriptors, two reviewers independently reviewed literature searches to identify potentially relevant trials for full review (BR, CS). From the full text, using specific criteria, two reviewers independently selected trials for inclusion in this review (KK, BR). Agreement was measured using kappa (к) statistics. Disagreement was resolved by consensus or third party adjudication (CS).

\section{Data extraction}

Data on study characteristics and outcome measures of efficiency and safety were extracted by two reviewers (KK, CS) using a standard form. Primary study authors were requested to confirm data extraction 
and provide additional clarification and information. Most authors could not access their original data to perform supplemental analyses. In some cases, expansion of graphic representations of data from the manuscripts was used to obtain estimates of outcomes.

\section{Assessment of methodological quality}

Two methods to assess methodological quality were used. Firstly, using the Cochrane approach to assessment of allocation concealment, all trials were scored as either: "adequate concealment", "uncertain", or "clearly inadequate concealment" [15]. In addition, each study was assessed using a $0-5$ scale that has been shown to be valid and reliable [16]. The scale examines issues and methods of randomization ( $0-2$ points) and double blinding (0-2 points), and descriptions of withdrawals and dropouts ( $0-1$ points). Inter-rater reliability was measured using $\kappa$ statistics [17]. Methodological quality assessments were performed by two reviewers working independently (KK, CS).

\section{Analysis}

All data were entered into and analysed using Review Manager (Version 4.0.1, MetaView 3.1, Update Software, Oxford, UK). The results are reported by outcome. The main results were reported as the weighted mean difference (WMD) in the maximum per cent fall in FEV1 between inhaled NCS and SCG. Other outcomes examined were complete protection, clinical protection, and side effects.

For dichotomous variables, individual and pooled statistics were calculated as odds ratios (OR) with 95\% confidence intervals $(95 \% \mathrm{CI})$; a random effects model was used. Because it is the convention in graphing meta-analytic results to have a point estimate which shows a benefit to an intervention fall on the left side of the midline (figs. 1-3), both complete and clinical protection data were analysed as a failure to achieve the event. NCS was considered the intervention, SCG

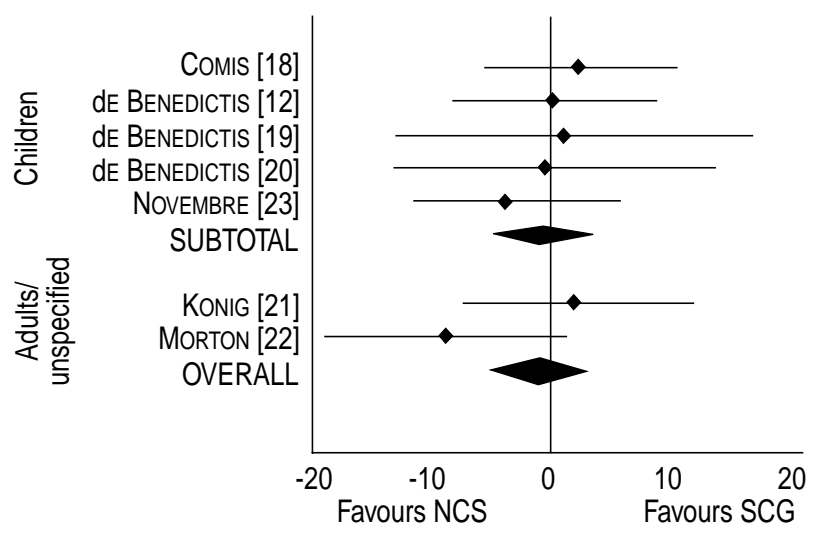

Fig. 1. - Nedocromil sodium (NCS) versus sodium cromoglycate (SCG) in the pretreatment of exercise induced bronchoconstriction; the maximum per cent decrease in forced expiratory volume in one second. Data are presented as weighted mean different (WMD) $(95 \%$ confidence intervals). WMD $=-0.88 \quad(-4.5$ 2.7); Chi-squared $=3.79$; degrees of freedom $=6 ; p=0.70$.

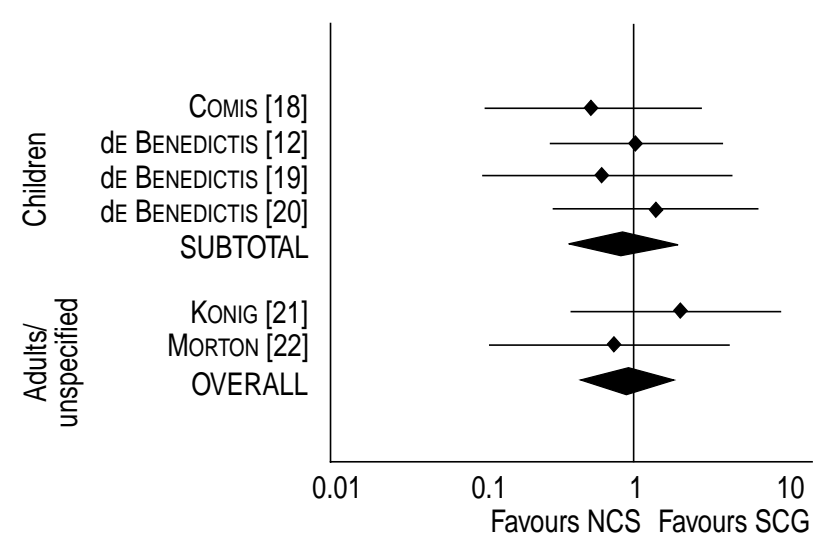

Fig. 2. - Nedocromil sodium (NCS) versus sodium cromoglycate (SCG) in the pretreatment of exercise induced bronchoconstriction; failure to achieve complete protection (maximum fall in forced expiratory volume in one second $(\mathrm{FEV} 1)$ remains $>10 \%)$. Data are presented as odds ratio (OR) (95\% confidence intervals). WMD: weighted mean differences. WMD $=-0.95 \quad(0.50-$ 1.81); Chi-squared $=1.88$; degrees of freedom $=5 ; \mathrm{p}=0.87$.

the control; therefore, point estimates to the left favour NCS and those to the right favour SCG. For continuous outcomes, individual and pooled statistics were calculated as WMD and 95\% CI using a random effects model. Two specific subgroup analyses were planned a priori. One was to compare adults $(\geqslant 18 \mathrm{yrs})$ with children (6-17 yrs). The other was to compare the various doses of NCS and SCG. Sensitivity analyses were conducted on fixed versus random effects and methodological quality (high versus low).

\section{Results}

\section{Search identification and characteristics}

A computerized search of the Asthma and Wheez* register yielded 106 citations of which $92(87 \%)$ were original publications. Search through 218,355 references in CCTR (1999, Version 1) resulted in five

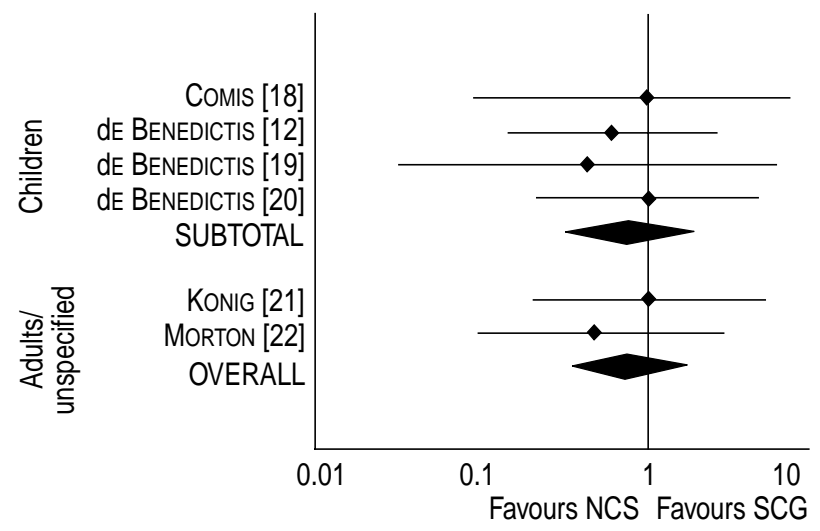

Fig. 3. - Nedocromil sodium (NCS) versus sodium cromoglycate (SCG) in the pretreatment of exercise induced bronchoconstriction; failure to achieve clinical protection $(<50 \%$ improvement over placebo). Data are presented as odds ratio (OR) $(95 \%$ confidence intervals). WMD: weighted mean differences. WMD = $-0.71(0.36-1.39)$; Chi-squared $=1.03$; degrees of freedom $=5$; $\mathrm{p}=0.96$. 
additional citations. Independent reviews of the abstracts and titles of these publications identified 14 potentially relevant studies. The agreement for relevance was excellent $(\kappa=0.90)$. Three potentially relevant citations were added from bibliographic searching of relevant articles and overviews. Independent review of these 17 potentially relevant articles determined that 8 trials met the inclusion criteria for this meta-analysis. The eight studies were published between 1987 and 1995. Five were from centres in Italy, and one each from Australia, the United Kingdom and the United States (table 1).

Participants. There were five studies conducted on children [12, 18-20, 23] two studies involving adults $[21,24]$ and one study that combined the age groups [22]. Since the mean age in this latter study was $20 \mathrm{yrs}$, it was allocated to the adult group. The majority of studies recruited stable asthmatics with a postexercise fall in FEV1 of $\geqslant 15 \%$. One study recruited EIB patients with a fall in FEV1 of $\geqslant 20 \%$ [21] (table 1).

Method. All studies incorporated a randomized, double blind, placebo-controlled, crossover study design. A pharmacological "carryover" effect was unlikely, since both NCS and SCG are topical short-acting agents that do not accumulate and both medications are rapidly cleared from the body [25]. In all studies, assessments were conducted with a minimum of $24 \mathrm{~h}$ elapsed time between challenges, to ensure drug clearance.

Interventions. Included trials evaluated a range of doses of both NCS and SCG, The doses for NCS ranged from $4 \mathrm{mg}[12,18-21,23,24]$ to $8 \mathrm{mg}$ [22], while the doses for SCG were $4 \mathrm{mg}$ [22], $10 \mathrm{mg}$ [12, 18-20, 23, 24] and $20 \mathrm{mg}$ [22]. Timing of administration varied as follows: $15 \mathrm{~min}$ [22], $20 \mathrm{~min}$ [12, 19-21, 23], $30 \mathrm{~min}$ $[18,24], 120 \mathrm{~min}[21], 140 \mathrm{~min}[20]$, and $240 \mathrm{~min} \mathrm{[22]}$ before a standardized exercise challenge (table 2). Exercise challenges were conducted on different days, at the same time of day, and in controlled indoor environments. Concomitant asthma medications were withheld for appropriate times prior to each of the challenges.

Outcomes. The most common outcome reported was the maximum per cent fall in FEV1 calculated as follows: ((pre-exercise baseline value - lowest postexer- cise value)/pre-exercise baseline value) $\times 100$. If the maximum per cent fall in FEV1 after treatment was $>15 \%[22,23]$ or $>10 \%[12,19-21]$ "complete protection" was not achieved. (In studies that reported individual patient data, data for both cut-points were calculated). If per cent fall after receiving the active drug was less than half the per cent drop after receiving placebo, "clinical protection" was not achieved. Side effects were reported in three studies [2123]. Other outcomes included: per cent fall in FEV1 at various time intervals, maximum per cent decrease in mean forced expiratory flow between when the lungs are 25 and $75 \%$ full (FEF25-75), per cent decrease in FEV1 multiplied by minutes (area under the curve), and maximum per cent decrease in PEFR. However, the number of studies reporting each of these measures was insufficient for pooling and these outcomes were not reported in detail. One study [24], which analysed only the change in per cent fall in FEV1 at different time points, was not included in the meta-analysis, and their findings are reported separately (table 2).

\section{Quality assessment of trials}

Overall, the agreement on quality between two independent reviewers was high, for the Cochrane approach, $\kappa=1.0$, and for the Jadad scale, $\kappa=0.89$. All studies were randomized and double blind. Only one study gave a description of withdrawals [22], otherwise there were no dropouts. Only one study described the method of randomization or the method of double blinding [24]. Using Jadad's 5-point validity scale, one study scored 5 [24], one scored 4 [22] and the remainder scored 3 . Using the Cochrane criteria to assess allocation concealment, one study was rated as having "adequate concealment" [24], and the remainder were rated as "uncertain". In general, the methodological quality of the included trials was rated as "moderate".

\section{Outcomes}

Pulmonary function results. Seven trials reported the maximum per cent fall in FEV1 as an outcome measure. The mean maximum fall in FEV1 reported in the trials ranged from $11.0-15.8 \%$ using NCS and from $9.8-24.1 \%$ using SCG when the exercise challenge was undertaken within $30 \mathrm{~min}$ of inhalation. On

Table 1. - Characteristics of included studies

\begin{tabular}{lccccrcc}
\hline Author [Ref.] & Location & Sample size & Population & Age & Male & $\begin{array}{c}\text { Female } \\
\text { Fall index } \\
\%\end{array}$ \\
\hline Comis [18] & Italy & 12 & $\mathrm{C}$ & $11(6.5-13.5)$ & 7 & 5 & 15 \\
DE BENEDICTIS [12] & Italy & 17 & $\mathrm{C}$ & $10.2 \pm 2.2(7-15)$ & 11 & 6 & 15 \\
De BENEDICTIS [19] & Italy & 8 & $\mathrm{C}$ & $8.7(7-11)$ & 5 & 3 & 15 \\
DE BENEDICTIS [20] & Italy & 13 & $\mathrm{C}$ & $10 \pm 2.3(7-15)$ & 9 & 4 & 15 \\
KonIG [21] & USA & 12 & $\mathrm{~A}$ & $27.3(21-38)$ & 12 & 0 & 20 \\
MoRTON [22] & Australia & 16 & $\mathrm{~B}$ & $20 \pm 4.8(13-30)$ & 10 & 6 & 15 \\
NovEMBRE [23] & Italy & 19 & $\mathrm{C}$ & $6-15$ & 13 & 6 & 15 \\
SINCLAIR [24] & UK & 20 & $\mathrm{~A}$ & $17-20.7$ & 18 & 2 & 15 \\
\hline
\end{tabular}

Data presented as $\mathrm{n}$, mean $\pm \mathrm{SD}$ (range), mean (range) or range. A: adult; C: child; B: both. 
Table 2. - Interventions and outcomes

\begin{tabular}{lccccc}
\hline Author [Ref.] & $\begin{array}{c}\text { Drug dose } \\
\text { versus placebo }\end{array}$ & $\begin{array}{c}\text { Time of therapy } \\
\text { pre-exercise } \\
\text { min }\end{array}$ & Major outcomes* & $\begin{array}{c}\text { Quality score } \\
\text { (Jadad) }\end{array}$ & $\begin{array}{c}\text { Comparison of } \\
\text { effectiveness }\end{array}$ \\
\hline COMIS [18] & $4 \mathrm{mg}$ NCS 10 mg SCG & 30 & $1,2,3$ & 3 & equal \\
DE BENEDICTIS [12] & $4 \mathrm{mg}$ NCS 10 mg SCG & 20 & $1,2,3,4$ & 3 & equal \\
DE BENEDICTIS [19] & $4 \mathrm{mg}$ NCS 10 mg SCG & 20 & $1,2,3,4$ & 3 & equal \\
DE BENEDICTIS [20] & $4 \mathrm{mg}$ NCS 10 mg SCG & 20,140 & $1,2,3,4,6$ & 3 & equal \\
KonIG [21] & $4 \mathrm{mg}$ NCS 20 mg SCG & $20,120,240$ & $1,2,4,5,6$ & 3 & equal \\
MoRTON [22] & 8 mg NCS 4 mg SCG & 15 & $1,2,3,5$ & 4 & equal \\
NovEMBRE [23] & $4 \mathrm{mg}$ NCS 10 m SCG & 20 & $1,3,5$ & 3 & uncertain \\
SiNCLAIR [24] & $4 \mathrm{mg}$ NCS 10 mg SCG & 30 & 7 & 5 & uncertain \\
\hline
\end{tabular}

*: $1=$ maximum per cent decrease in forced expiratory volume in one second, $2=$ complete protection $(<10 \%), 3=$ complete protection $(<15 \%), 4=$ clinical protection; $5=$ side effects; $6=$ duration; $7=$ other.

pooling these results, no significant difference between NCS and SCG with respect to the maximum per cent decrease in FEV1 was noted (WMD=-0.88; 95\% CI 4.49-2.74). No statistically significant heterogeneity was identified (Chi-squared $=3.79$; degrees of freedom= 6; $p>0.70$ ) (fig. 1).

In the two trials that examined effects at $120-140$ min post inhalation [20, 21], the mean maximum fall FEV1 reported in the trials ranged from $23.1-23.9 \%$ using NCS and from 16.8-21.9\% using SCG. Again, there was no significant difference between the drugs (WMD $=5.0 ; 95 \%$ CI -3.01-13.01) although the point estimate favoured SCG.

One study [24], was not included in the pooled result because it reported only the per cent changes from baseline FEV1 as the outcome measure. This study supports the findings of the other seven trials in that $4 \mathrm{mg}$ of NCS was equivalent to $10 \mathrm{mg}$ of $\mathrm{SCG}$ in preventing exercise-induced bronchoconstriction.

Complete protection. Seven trials reported complete protection as an outcome measure. No significant difference between NCS and SCG with respect to complete protection was evident during the study period. When failing to achieve complete protection was defined as the maximum fall in FEV1 remained $>10 \%(n=6), 53 \%$ taking NCS and 54\% taking SCG did not reach this goal $(\mathrm{OR}=0.95 ; 95 \% \mathrm{CI}$ : $0.50-1.81$; fig. 2). When defined as the maximum fall in FEV1 remained $>15 \%(n=6), 36 \%$ taking $\mathrm{NCS}$ and $46 \%$ taking SCG did not achieve complete protection $(\mathrm{OR}=0.69 ; 95 \% \mathrm{CI}$ : $0.33-1.47)$. Following pooling of these studies, no statistically significant heterogeneity was noted (Chi-squared $=1.88$; degrees of freedom $=5$; $\mathrm{p}=0.87$ ) and (Chi-squared $=6.77$; degrees of freedom= $5 ; \mathrm{p}=0.24)$, respectively.

In the two trials that examined effects at 120 $140 \mathrm{~min}$ post inhalation [20,21], 76\% taking NCS and $64 \%$ taking SCG failed to achieve complete protection $(>10 \%), \mathrm{OR}=1.78 ; 95 \% \mathrm{CI}: 0.52-6.09$. At 240 min post inhalation [21], these figures rose to $83 \%$ $(\mathrm{NCS})$ and $75 \%(\mathrm{SCG})(\mathrm{OR}=1.67 ; 95 \% \mathrm{CI}: 0.22-12.35)$.

Clinical protection. Six trials ( $n=4$ children, $n=2$ adult) reported clinical protection as an outcome measure. There was no significant difference between NCS and SCG with respect to clinical protection; $33 \%$ receiving
NCS and $41 \%$ receiving SCG failed to obtain at least a $50 \%$ improvement in FEV1 over placebo $(\mathrm{OR}=0.71$; 95\% CI: 0.36-1.39). When these studies were pooled, no statistically significant heterogeneity was demonstrated (Chi-squared $=1.03$; degrees of freedom $=5 ; p=0.96$; fig. 3).

In the two trials that examined effects at 120 140 min post inhalation $[20,21], 84 \%$ receiving NCS and $60 \%$ receiving SCG failed to achieve clinical protection $(\mathrm{OR}=3.30 ; 95 \% \mathrm{CI} 0.83-13.11)$. At $240 \mathrm{~min}$ post inhalation [21] 83\% (NCS) and 67\% (SCG) did not receive clinical protection $(\mathrm{OR}=2.50 ; 95 \% \mathrm{CI} 0.36-17.32)$.

Side effects. Three trials reported side effects as an outcome measure $(n=3$, unpleasant taste, $n=2$ sore throat). No significant difference was noted between NCS and SCG with respect to unpleasant taste $(\mathrm{OR}=6.85$; 95\% CI: 0.77-60.73). No statistically significant heterogeneity existed when these studies were pooled (chi-squared $=2.78$; degrees of freedom $=1 ; \mathrm{p}=$ $0.10)$. In addition, no significant difference between NCS and SCG was noted for sore throat $(\mathrm{OR}=3.46$; 95\% CI 0.32-37.48) in the two studies that measured this outcome. Overall, the trend in both cases was for fewer side effects in the SCG group. The number of trials reporting side effects was small; hence, the lack of significance may be an effect of the lack of statistical power.

\section{Subgroup/sensitivity analyses}

Subgroup analyses that were based on age suggest similar results for children and adults for maximum per cent decrease in FEV1, complete protection and clinical protection.

Sensitivity analyses were performed using various doses of both NCS and SCG, and timing of exercising postinhalation. The results were consistent with the pooled analyses. No statistically significant differences between the two medications, using various dosages (NCS $4 \mathrm{mg}$ or $8 \mathrm{mg}$, and SCG $4 \mathrm{mg}, 10 \mathrm{mg}$ or $20 \mathrm{mg}$ ), with regards to maximum per cent decrease in FEV1, complete protection and clinical protection were identified. Also, timing of exercise postinhalation $(\leqslant 30 \mathrm{~min}$, $120-140 \mathrm{~min}$, or $240 \mathrm{~min}$ ) did not affect the difference between the two medications with regards to maximum per cent decrease in FEV1, complete protection 
or clinical protection. Both drugs exhibited reduced efficacy over time but again, the number of trials involved were so few that any true difference between them may not have been detected.

The results are presented using random effects modelling. Analyses using fixed effects modelling yielded similar results, although fixed effects models generated narrower confidence intervals. Pertaining to side effects, although the point estimation remained consistent, the narrower confidence interval as a consequence of the use of a fixed effect model resulted in SCG having significantly fewer side effects.

Since all studies were rated as moderate quality (Jadad score $=3-5$ ), sensitivity analysis based on quality rating was not conducted.

\section{Discussion}

This systematic review compared the effects of NCS and SCG administered by a pressurized aerosol prior to a strenuous exercise challenge in participants with EIB. The pooled result failed to demonstrate any significant difference between the effect of these two medications on pulmonary function - specifically maximum per cent decrease in FEV1, the drugs' ability to provide complete protection or clinical protection, or side effects at any time postinhalation. Adverse effects were mild and the meta-analysis failed to demonstrate superiority of either medication in the doses studied. No significant heterogeneity was evident among the pooled results, indicating that the trials were pooled appropriately and that the results of the included trials are homogeneous. Similarly, subgroup analyses based on age, and sensitivity analyses taking into consideration various doses of the two medications and timing of exercise postinhalation, consistently found a nonsignificant difference between these two medications. Therefore, both NCS and SCG inhaled prior to strenuous physical exercise appear similarly efficacious in preventing deterioration in lung function during the immediate postexercise period in adults and children with EIB. Efficacy decreased over time but no significant differences between the two were evident in challenges taken $2-4 \mathrm{~h}$ postinhalation.

There are several clinical implications of this review. In general, clinicians and patients can be reassured that these agents may be equally helpful in treating EIB with little risk of major side effects. Both medications significantly attenuate the EIB response and in approximately $50 \%$ of patients are able to provide complete protection; however, no specific recommendations regarding the preferred medication can be made. Unfortunately, no data reporting, symptom scores, or participant preference were available.

Since both NCS and SCG function to "prevent" rather than treat EIB when its symptoms occur, the option to pretreat may be attractive to both patients and physicians. Overall, given the results of this review, these agents should be added to the treatment armamentarium for patients on an individualized basis.

As with any systematic review, certain limitations of the review may exist [26, 27], and the possibility of publication and selection biases should be addressed. A comprehensive search of published literature for potentially relevant studies was conducted, and attempts were made to contact corresponding and first authors to identify unpublished work. Publication bias often exists when negative trials, indicating no significant differences between drugs, are not published and therefore not included in a review. Considering that the results of all the trials in this review independently illustrated no significant difference between the two medications, the identification of further negative trials should not alter this conclusion. Though it is possible that a selection bias occurred, this review employed two independent reviewers for the selection process, and the authors are confident that the studies excluded were done so for appropriate reasons and in a consistent manner.

All participants in the included studies had stable asthma, and experienced a wide range of severity of EIB on control and placebo runs. Therefore, the results of this review may be generalized to all stable asthmatics with EIB. Nonetheless, further research should focus on dose comparisons, measuring the effects of NCS and SCG on other clinical outcomes such as quality of life, symptom scores, patient preference and comparing these agents to other EIB medications (such as salbutamol, leukotriene modifiers, inhaled steroids, etc.).

In summary, no differences in efficacy in exerciseinduced bronchoconstriction treatment were demonstrated in the comparison of nedocromil sodium and sodium cromoglycate. Although the analyses of side effects favour sodium cromoglycate, none of the side effects exhibited were major. The costs for both treatments are similar, and both agents are easy to administer and sensible to use. Therefore, both these agents should be considered for treatment of exerciseinduced bronchoconstriction.

Acknowledgements. The authors wish to acknowledge the assistance of S. Milan, A. Bara and J. Dennis of the Cochrane Airways Review Group. The authors would also like to acknowledge the assistance of the following corresponding authors: F.M. de Benedictis, D.G. Sinclair and A. Comis. Finally, the assistance of P. Jones (Airways Review Group Editor) in the reviewing the manuscript was greatly appreciated.

In terms of authors contributions, K.D. Kelly was responsible for most of the project management, quality assessment, data extraction and preparation of the manuscript. C.H. Spooner was involved in assessment of trials for inclusion, quality assessment, data extraction, and editorial review of the paper. B.H. Rowe helped assess the trials for inclusion, checked the data for accuracy, provided methodological support, and editorial review of the paper. B.H. Rowe was the Airways Review Group's Assigned Editor for this review.

\section{References}

1. Anderson SD. Issues in exercise-induced asthma. J Allergy Clin Immunol 1985; 76: 763-772.

2. Spector SL. Update on exercise-induced asthma. Ann Allergy 1993; 71: 571-577. 
3. Bar-Yishay E, Godfrey S. Mechanisms of exerciseinduced asthma. Lung 1984; 162: 851-855.

4. Eggleston PA. Methods of exercise challenge. J Allergy Clin Immunol 1994; 73: 666-669.

5. ERS Task Force. Clinical exercise testing with reference to lung diseases: indications, standardization and interpretation strategies. Eur Respir J 1997; 10: 2662-2689.

6. Virant FS. Exercise-induced bronchospasm: epidemiology, pathophysioiogy, and therapy. Med Sci Sports Exerc 1992; 24: 851-855.

7. Rupp NT. Diagnosis and management of exerciseinduced asthma. Phys Sportsmed 1996; 24: 77-87.

8. Sly RM. Beta-adrenergic drugs in the management of asthma in athletes. J Allergy Clin Immunol 1984; 73: 680-685.

9. Spooner CA, Saunders D, Rowe BH. Systematic review of Nedocromil Sodium in exercise induced bronchoconstriction (Cochrane Review), Cochrane Library, Issue 3, (1998) Oxford: Update Software.

10. Drugs for asthma. The Medical Letter 1999; 41 (1044): 5-10.

11. Holgate ST. Clinical evaluation of nedocromil sodium in asthma. Eur J Respir Dis 1986; 69: 149-159.

12. de Benedictis FM, Tuteri G, Bertotto A, Bruni L, Vaccaro R. Comparison of the protective effects of cromolyn sodium and nedocromil sodium in the treatment of exercise-induced asthma in children. J Allergy Clin Immunol 1994a; 94: 684-688.

13. Anderson SD. Specific problems: exercise-induced asthma. In: O'Byrne P, Thompson NC, eds. Manual of Asthma Management. London, W.B. Saunders Co., 1995; pp. 621-643.

14. Dickersin K, Scherer R, Lefebvre C. Identifying relevant studies for systematic reviews. BMJ 1994; 309: 1286-1291.

15. Mulrow CD, Oxman AD. Cochrane Collaboration Handbook (updated September 1997). The Cochrane Library (database on disk on CDROM) Issue 1. 1994. Oxford, UK, Update Software.

16. Jadad AR, Moore RA, Carroll D, et al. Assessing the quality of reports of randomized clinical trials: Is blinding necessary? Control Clin Trials 1996; 17: 1-12.
17. Kramer MS, Feinstein AR. Clinical biostatistics LIV. The biostatistics of concordance. Clin Pharmacol Ther 1981; 29: 111-123.

18. Comis A, Valetta EA, Sette L, Andreoli A, Boner AL. Comparison of nedocromil sodium and sodium cromoglycate administered by pressurized aerosol, with and without a spacer device in exercise-induced asthma in children. Eur Respir $J$ 1993; 6: 523-526.

19. de Benedictis FM, Tuteri G, Niccoli A, Mezzetti D, Rossi L, Bruni L. The effect of cromolyn sodium and nedocromil sodium administered by a pressurized aerosol with a spacer device on exercise-induced asthma in children. Mediat Inflamm 1994; 3: S35-S37.

20. de Benedictis FM, Tuteri G, Pazzelli P, Bertotto A, Bruni L, Vaccaro R. Cromolyn versus nedocromil: duration of action in exercise-induced asthma in children. J Allergy Clin Immunol 1995; 96: 510-514.

21. Konig P, Hordvik NL, Kreutz C. The preventive effect and duration of action of nedocromil sodium and cromolyn sodium on exercise-induced asthma (EIA) in adults. J Allergy Clin Immunol 1987; 79: 64-68.

22. Morton AR, Ogle SL, Fitch KD. Effects of nedocromil sodium, cromolyn sodium, and a placebo in exerciseinduced asthma. Ann Allergy 1992; 68: 143-148.

23. Novembre E, Frongia GF, Veneruso G, Vierucci A. Inhibition of exercise-induced asthma (EIA) by nedocromil sodium and sodium cromoglycate in children. Pediatr Allergy Immunol 1994; 5: 107-110.

24. Sinclair DG, Winfield CR. Attenuation of exercise induced asthma by nedocromil sodium and sodium cromoglycate. J Royal Army Med Corps 1990; 136: 105-106.

25. CPS. Compendium of Pharmaceuticals and Specialties. 34th ed. Ottawa, Canadian Pharmacists Association 1999.

26. Oxman AD, Cook DJ, Guyatt GH, for the EvidenceBased medicine Working Group Users' guides to the medical literature VI. How to use an overview. JAMA 1994; 272: 1367-1371.

27. Oxman AD, Guyatt GH. Guidelines for reading literature reviews. Can Med Assoc J 1988; 138: 697703. 